Journal of Mathematics and Statistics 1 (3): 246-251, 2005

ISSN 1549-3644

(c) 2005 Science Publications

\title{
Numerical Ultimate Ruin Probabilities under Interest Force
}

\author{
${ }^{1}$ Juma Kasozi and ${ }^{2}$ Jostein Paulsen \\ ${ }^{1}$ Department of Mathematics, Makerere University, Box 7062, Kampala, Uganda \\ ${ }^{2}$ Department of Mathematics, University of Bergen, 5008 Bergen, Norway
}

\begin{abstract}
This work addresses the issue of ruin of an insurer whose portfolio is exposed to insurance risk arising from the classical surplus process. Availability of a positive interest rate in the financial world forces the insurer to invest into a risk free asset. We derive a linear Volterra integral equation of the second kind and apply an order four Block-by-block method in conjuction with the Simpson rule to solve the Volterra equation for ultimate ruin. This probability is arrived at by taking a linear combination of some two solutions to the Volterra integral equation. The several numerical examples given show that our results are excellent and reliable.
\end{abstract}

Key words: Risk theory, ruin probability, volterra integral equation, block-by-block method

\section{INTRODUCTION}

The problem of finding the probability of ultimate ruin was first considered by Lundberg ${ }^{[1]}$. Since then, the problem has received much attention up to present day. In his thesis, Lundberg considered a surplus model of the type:

Surplus $=$ Initial reserve + Income - outflow. Among the earlier authors who gave a rigorous mathematical basis of Lundberg's work was Cramér ${ }^{[2,3]}$. His contributions were presented in his monograph 'Collective Risk Theory'. Lundberg's model, expounded by Cramér, is termed the Cramér-Lundberg model or the surplus model.

In this model, at time $t$, the surplus $Y_{t}$ of an insurance company is given by

$Y_{t}=y+p t-\sum_{i=1}^{N_{t}} S_{i}$

where, $y=Y_{0} \geq 0$ is the initial reserve, $\mathrm{p}>0$ is the premium rate, $\left\{N_{t}\right\}_{t \in \mathfrak{R}^{+}}$is a Poisson process with intensity $\lambda$, modelling the number of claims in $(0, \mathrm{t}]$ and $\left\{S_{i}\right\}_{i \in \mathrm{N}}$ is an independent and identically distributed sequence of positive random variables (with distribution $F$ ) independent of $\mathrm{N}$, modelling the claim sizes. The distribution $F$ has finite expectation $\mu$ and finite variance $\sigma^{2}$. In the literature, the process $\mathrm{Y}$ in equation (1) is commonly known as the classical risk model.

A critical look at the process in (1) raises a couple of questions. One question that has received much attention is 'what is the probability that $\mathrm{Y}$ ever becomes negative?' The first time when this happens is termed the time of ruin and the associated probability is the probability of ruin. Ruin is considered as a technical term. It does not mean that the company is bankrupt. However, if ruin occurs, it is interpreted as meaning that the company has to take action in order to make the business profitable.

The Cramér-Lundberg model serves as a skeleton for more realistic models that have been studied in the insurance literature. This standard model for nonlife insurance is simple enough to calculate probabilities of interest, but too simple to be realistic. For example, it does not include interest earned on the invested surplus. There are several papers treating this model in many directions and forms, all with a view of finding the probability of ruin. By far the majority of these papers are concentrated on the analytical aspects of the problem but there is also a quite considerable number that deal with numerical methods to calculate this probability. More on the history of this problem can be traced from Segerdahl ${ }^{[4,5]}$, Andersen ${ }^{[6]}$, Davidson ${ }^{[7]}$, Thorin $^{[8]}$, Wikstad ${ }^{[9]}$, Gerber ${ }^{[10]}$, Harrison ${ }^{[11]}$, De Vylder $^{[12]}$. For a general background to ruin theory, we refer to Bühlmann ${ }^{[13]}$. In this study, we shall be concerned with ruin under interest force and our emphasis will be on numerical methods.

\section{THE MODEL AND THEORETICAL RESULTS}

All processes and random variables are assumed to be defined on the stochastic basis $\left(\Omega, f,\left\{f_{t}\right\}_{t \in \mathfrak{R}^{+}, \mathrm{P}}\right)$ satisfying the usual conditions, i.e. $f_{t}$ is right continuous and P-complete. Here, $\Omega$ is an abstract sample space whose elements are denoted as $\omega ; f$ is a $\sigma$-algebra on $\Omega ; \mathrm{P}$ is a probability measure and $\left\{f_{t}\right\}_{t \in \mathfrak{R}^{+}}$is a filtration. A filtration means an increasing and right continuous family of sub $\sigma$ -

Corresponding Author: Juma Kasozi, Department of Mathematics, Makerere University, P.O. Box 7062, Kampala, Uganda 
algebras of $f$, that is, for $0 \leq s \leq t, f_{s} \subset f_{t} \subset f$ and $f_{t}=f_{t+}=\cap_{s>t} f_{s}$.

The risk model for the surplus $Y_{t}$ of an insurance company at time $t$ is given by equation (1). A natural generalisation of this model is to allow reserves to earn interests. If there is a positive constant force of interest $r$, the surplus now become

$$
\begin{aligned}
& Y_{t}=y+p t+r \int_{0}^{t} Y_{s} d s-\sum_{i=1}^{N_{t}} S_{i} \\
& \quad \text { Let } \psi(y)=\mathrm{P}\left(\mathrm{T}_{y}<\infty\right) \text { where } \mathrm{T}_{y} \text { is the time of }
\end{aligned}
$$
ruin, equal to infinity if ruin never occurs. Then $\psi(y)$ is the probability of ultimate ruin when initial capital is $\mathrm{y}$ and it is well known ${ }^{[14]}$ that satisfies the integrodifferential equation

$$
(r y+p) \psi^{\prime}(y)+\lambda \int_{0}^{\infty}(\psi(y-x)-\psi(y)) d F(x)=0 .
$$

Obviously $\psi(y)=1$ for $\mathrm{y}<0$ and it is well known

[15] Theorem 3.1, that $\lim _{y \rightarrow \infty} \psi(y)=0$. Using integration by parts, this equation is easily transformed into the Volterra equation of the second kind

$$
\begin{aligned}
& \psi(\mathrm{y})=\frac{\mathrm{p}}{\mathrm{ry}+\mathrm{p}} \psi(0)-\frac{\lambda}{\mathrm{ry}+\mathrm{p}} \\
& \int_{0}^{\mathrm{y}} \overline{\mathrm{F}}(\mathrm{x}) \mathrm{dx}+\int_{0}^{\mathrm{y}} \mathrm{K}(\mathrm{y}, \mathrm{x}) \psi(\mathrm{x}) \mathrm{dx},
\end{aligned}
$$

where the kernel

$$
K(y, x)=\frac{r+\lambda \bar{F}(y-x)}{r y-p}
$$

and $\bar{F}(x)=1-F(x)$.

If we knew $\psi(0)$, (2) could be solved using some numerical method for Volterra equations. For the classical risk model, $\psi(0)=\frac{\lambda \mu}{p}$ when $\lambda \mu<p$ and $\psi(0)=1$ otherwise. An expression for $\psi(0)$ when $r>0$ is provided in equation (14) in Sundt and Teugels ${ }^{[16]}$, but this is rather complicated to calculate, also numerically. Sundt and Teugels give easy to calculate inequalities for $\psi(0)$, but they are less useful if one is interested in exact values for $\psi(y)$. Asmussen and Nielsen ${ }^{[17]}$ provide an efficient simulation procedure for $\psi(y)$, but that can be a bit complicated to implement and it works only when $\lambda \mu<p$ and the moment generating function $\mathrm{E}\left[e^{u S}\right]$ exists for some positive u. Simpler simulation procedures are suggested in Paulsen and Rasmussen ${ }^{[18]}$, but they are less precise than those of Asmussen and Nielsen. They also do not work well when $\lambda \mu \geq p$. The method we present in this work works well $\lambda \mu \geq p$.
We will now show how equation (2) can be used to find $\psi(y)$. To this end, let $g_{a}(y)$ be the solution of

$$
\begin{aligned}
& g_{a}(y)=\frac{p}{r y+p} a-\frac{\lambda}{r y+p} \int_{0}^{y} \bar{F}(x) d x+ \\
& \int_{0}^{y} K(y, x) g_{a}(x) d x,
\end{aligned}
$$

where, $K(y, x)$ is as in (3). Note that in particular $g_{\psi(0)}(y)=\psi(y)$. It follows by Linz ${ }^{[19]}$, Theorem 3.2 p.32 and dominated convergence that (4) has a unique continuous solution for all $\mathrm{y}$ and all a. The following result will be proved at the end.

Theorem 1: For all $a$, the equation (4) has a unique continuous and bounded solution and $g_{a}(\infty)=\lim _{y \rightarrow \infty} g_{a}(y)$ exists.

Let $a_{1} \neq a_{2}$ and set

$$
\tilde{g}_{\alpha, a_{1}, a_{2}}(y)=\alpha g_{a_{1}}(y)+(1-\alpha) g_{a_{2}}(y)
$$

Then $\tilde{g}_{\alpha, a_{1}, a_{2}}$ also satisfies equation (4) with $\tilde{g}_{\alpha, a_{1}, a_{2}}(0)=\alpha a_{1}+(1-\alpha) a_{2}$. From this it is clear that any solution of (4) can be written in the form (5) for a suitable choice of $\alpha$. In particular, the choice

$$
\alpha=\frac{\psi(0)-a_{2}}{a_{1}-a_{2}}
$$

gives $\tilde{g}_{\alpha, a_{1}, a_{2}}(0)=\psi(0)$ and therefore by uniqueness of the solution, $\tilde{g}_{\alpha, a_{1}, a_{2}}(y)=\psi(y)$. But with this $\alpha, \quad \alpha g_{a_{1}}(\infty)+(1-\alpha) g_{a_{2}}(\infty)=\psi(\infty)=0$, hence,

$$
\alpha=\frac{g_{a_{2}}(\infty)}{g_{a_{2}}(\infty)-g_{a_{1}}(\infty)}
$$

We thus have

Corollary: Let $a_{1} \neq a_{2}$ and $\alpha$ be given by (6). Then $\psi(y)=\alpha g_{a_{1}}(y)+(1-\alpha) g_{a_{2}}(y)$,

The procedure to numerically find $\psi(y)$ is now straightforward. To solve (4) for two starting values $a_{1}$ and $a_{2}$, we use a numerical method like the block-byblock that we describe later. Run them until they have stabilised at (or very near) $g_{a_{1}}(\infty)$ and $g_{a_{2}}(\infty)$. Then calculate $\psi(y)$ according to (7). In the sequel and without loss of generality, $a_{2}=1$. 
Now to the proof of Theorem 1. Letting

$h_{a}(y)=g_{a}(y)-\psi(y)$,

(2) and (4) give

$h_{a}(y)=\frac{p}{r y+p} h_{a}(0)+\int_{0}^{y} K(y, x) h_{a}(x) d x$

where, of course $h_{a}(0)=a-\psi(0)$.

Lemma 1: The absolute value $\left|h_{a}(y)\right|$ is increasing in $y$.

Proof: Formal differentiation in (8) gives

$h_{a}^{\prime}(y)=\frac{\lambda}{r y+p}\left(h_{a}(0) \bar{F}(y)+\int_{0}^{y} \bar{F}(x) h_{a}^{\prime}(y-x) d x\right)$.

Actually, considering $\lim _{\delta \rightarrow 0} \frac{h_{a}(y+\delta)-h_{a}(y)}{\delta}$ shows that this equation is correct for $h_{a}^{+}(y)=\frac{d^{+}}{d y} h_{a}(y)$, i.e

$\mathrm{h}_{\mathrm{a}}^{+}(\mathrm{y})=\frac{\lambda}{\mathrm{ry}+\mathrm{p}}$

$\left(\mathrm{h}_{\mathrm{a}}(0) \overline{\mathrm{F}}(\mathrm{y})+\int_{0}^{\mathrm{y}} \overline{\mathrm{F}}(\mathrm{x}) \mathrm{h}_{\mathrm{a}}^{+}(\mathrm{y}-\mathrm{x}) \mathrm{dx}\right)$.

By Moiseiwitsch ${ }^{[20]}$, (9) has a unique integrable solution on any interval $(0, \mathrm{~T}]$.

Letting $\mathrm{y}=0$ in (9) gives $h_{a}^{+}(0)=\frac{\lambda}{p} h_{a}^{+}(0)$, hence $h_{a}^{+}(0)$ has the same sign as $h_{a}(0)$. If we assume that $y_{0}=\inf \left\{y: h_{a}^{+}(y)\right.$ has opposite sign of $\left.h_{a}^{+}(0)\right\}$ is finite, it follows from (9) that this leads to a contradiction, hence $y_{0}=\infty$. The result follows.

Proof of Theorem 1: By Lemma 1, $\lim _{y \rightarrow \infty} g_{a}(y)=\lim _{y \rightarrow \infty} h_{a}(y) \quad($ since $\psi(\infty)=0)$ exists, but may be infinite. Again by Lemma 1, to prove that the limit is finite, it is sufficient to prove that

$\int_{0}^{y}\left|h_{a}(x)\right| d x \leq C y(1+\varepsilon(y))$

for some constant $C$ and a function $\varepsilon$ which satisfies $\lim _{y \rightarrow \infty} \varepsilon(y)=0$. Assume first that $h_{a}(0) \geq 0$, hence by Lemma $1, h_{a}(y) \geq 0$ for all y. By the version of a Tauberian theorem given in Linz ${ }^{[19]}$, Theorem 6.8, (10) follows provided we can prove that $\lim _{t \rightarrow \infty} t L_{h_{a}}(t)=C$, where, $\quad L_{h_{a}}(t)=\int_{0}^{\infty} e^{-t y} h_{a}(y) d y$ is the Laplace transform of $h_{a}$. By Linz $^{[19]}$, $h_{a}(y) \leq h_{a}(0) \exp \left\{\frac{r+\lambda}{p} y\right\}$, hence $L_{h_{a}}(t)$ exists at least for $t>\frac{r+\lambda}{p}$. Taking the Laplace transform for $t>\frac{r+\lambda}{p}$ on both sides of (8) gives after a few calculations,

$L_{h_{a}}^{\prime}(t)+\left(\frac{1}{t}+\frac{\lambda}{r} L_{\bar{F}}(t)-\frac{p}{r}\right) L_{h_{a}}(t)=-\frac{p h_{a}(0)}{r} \frac{1}{t}$,

where

$$
L_{\bar{F}}(t)=\int_{0}^{\infty} e^{-t y} \bar{F}(y) d y
$$

Note that $L_{\bar{F}}(0)=\mu$ and consequently $L_{\bar{F}}(z)$ is analytic for $\operatorname{Re}(\mathrm{z})>0$. Solving (11) gives

$\mathrm{L}_{\mathrm{h}_{\mathrm{a}}}(\mathrm{t})=\frac{1}{\mathrm{t}} \exp \left(\frac{\mathrm{p}}{\mathrm{r}} \mathrm{t}-\frac{\lambda}{\mathrm{r}} \int_{\mathrm{t}}^{\mathrm{c}_{1}} \mathrm{~L}_{\overline{\mathrm{F}}}(\mathrm{u}) \mathrm{du}\right)$
$=\left(\mathrm{c}_{3}+\frac{\mathrm{ph}}{\mathrm{r}}(0) \int_{\mathrm{t}}^{c_{2}} \exp \left(-\frac{\mathrm{p}}{\mathrm{r}} \mathrm{u}+\frac{\lambda}{\mathrm{r}} \int_{\mathrm{u}}^{c_{1}} \mathrm{~L}_{\overline{\mathrm{F}}}(\mathrm{s}) \mathrm{ds}\right) \mathrm{du}\right)$,

Where, $c_{1}, c_{2}$ and $c_{3}$ are constants. Replacing $\mathrm{t}$ by a complex $\mathrm{z}$ in (12), it is clear that the right hand side of (12) is analytical for $\operatorname{Re}(\mathrm{z})>0$. Hence, by Widder $^{[21]}, L_{h_{a}}(t)$ exists for all $\mathrm{t}>0$. Furthermore

$\lim _{t \rightarrow 0} t L_{h_{a}}(t)=\exp \left(-\frac{\lambda}{r} \int_{0}^{c} 1 L_{\bar{F}}(u) d u\right)$

$=\left(c_{3}+\frac{\mathrm{ph}_{\mathrm{a}}(0)}{\mathrm{r}} \int_{0}^{\mathrm{c}_{2}} \exp \left(-\frac{\mathrm{p}}{\mathrm{r}} \mathrm{u}+\frac{\lambda}{\mathrm{r}} \int_{\mathrm{u}}^{\mathrm{c}_{1}} \mathrm{~L}_{\overline{\mathrm{F}}}(\mathrm{s}) \mathrm{ds}\right) \mathrm{du}\right)$

$=C<\infty$

If $a-\psi(0)<0$, simply let $\hat{h}_{a}(y)=-h_{a}(y)$.

Then $\hat{h}_{a}(y)$ satisfies (8) with $\hat{h}_{a}(0)>0$. This ends the proof of the theorem.

\section{NUMERICAL METHODS}

Here, we will discuss numerical solutions of (2) (and its associated equation (4)).

To fix ideas, we write a general form of both equations thus

$g(y)+\int_{0}^{y} K(y, x) g(x) d x=\beta(y)$

Where, $K(y, x)$ is as in (3) and the expression for the forcing function $\beta(y)$ is obvious. Using a fixed grid $y=0, h, 2 h, \ldots$, the solution of (13) is of the form

$g_{n}+h \sum_{i=1}^{n} \omega_{i} K_{n, i} g_{i}=\beta_{n}$ 
where, $g_{i}$ is the numerical approximation to $g(i h), \quad K_{n, i}=K(n h, i h), g_{n}=g(n h) \quad$ and $\beta_{n}=\beta(n h)$. The $\omega_{i}$ are the integration weights. Here we shall use the block-by-block method in conjuction with the Simpson integration rule, thus obtaining solutions in blocks of 2 . The block-by-block is recommended $^{[22]}$ as the best of the higher order methods for numerically solving (13). To briefly explain the method, Simpson's rule gives

$g_{2}+\frac{h}{3}\left(K_{2,0} g_{0}+4 K_{2,1 g_{1}}+K_{2,2 g_{2}}\right)=\beta_{2}$

Here $g_{1}$ is unknown, but using the same rule with stepsize $\frac{h}{2}$, Simpson's rule gives

$g_{1}+\frac{h}{6}\left(K_{1,0} g_{0}+4 K_{1, \frac{1}{2} g} \frac{1}{2}+K_{1,1 g_{1}}\right)=\beta_{1}$

Quadratic interpolation gives that $g_{\frac{1}{2}} \approx \frac{3}{8} g_{0}+\frac{3}{4} g_{1}-\frac{1}{8} g_{2}$ and inserting this into (16) yields

$\mathrm{g}_{1}+\frac{\mathrm{h}}{6}\left(\begin{array}{l}\left(\mathrm{K}_{1,0}+\frac{3}{2} \mathrm{~K}_{1, \frac{1}{2}}\right) \mathrm{g}_{0}+ \\ \left(\mathrm{K}_{1,1}+3 \mathrm{~K}_{1, \frac{1}{2}}\right) \mathrm{g}_{1}-\frac{1}{2} \mathrm{~K}_{1, \frac{1}{2}} \mathrm{~g}_{2}\end{array}\right)=\beta_{1}$

Equations (15) and (17) is a pair of equations to solve for $g_{1}$ and $g_{2}$. Continuing like this in blocks of 2 we get,

$\mathrm{g}_{2 \mathrm{~m}+2}+\mathrm{h} \sum_{\mathrm{i}=0}^{2 \mathrm{~m}} \omega_{\mathrm{i}} \mathrm{K}_{2 \mathrm{~m}+2, \mathrm{~g}} \mathrm{~g}_{\mathrm{i}}+$

$\frac{\mathrm{h}}{3}\left(\begin{array}{l}\mathrm{K}_{2 \mathrm{~m}+2,2 \mathrm{~m}} \mathrm{~g}_{2 \mathrm{~m}}+4 \mathrm{~K}_{2 \mathrm{~m}+2,2 \mathrm{~m}+1} \mathrm{~g}_{2 \mathrm{~m}+1} \\ +\mathrm{K}_{2 \mathrm{~m}+2,2 \mathrm{~m}+2} \mathrm{~g}_{2 \mathrm{~m}+2}\end{array}\right)=\beta_{2 \mathrm{~m}+2}$

and

$\mathrm{g}_{2 \mathrm{~m}+1}+\mathrm{h} \sum_{\mathrm{i}=0}^{2 \mathrm{~m}} \omega_{\mathrm{i}} \mathrm{K}_{2 \mathrm{~m}+1, \mathrm{i}} \mathrm{g}_{\mathrm{i}}+$

$\frac{\mathrm{h}}{6}\left(\begin{array}{l}\mathrm{K}_{2 \mathrm{~m}+1,2 \mathrm{~m}} \mathrm{~g}_{2 \mathrm{~m}}+4 \mathrm{~K}_{2 \mathrm{~m}+1,2 \mathrm{~m}+\frac{1}{2}} \mathrm{~g}_{2 \mathrm{~m}+\frac{1}{2}} \\ +\mathrm{K}_{2 \mathrm{~m}+1,2 \mathrm{~m}+1} \mathrm{~g}_{2 \mathrm{~m}+1}\end{array}\right)=\beta_{2 \mathrm{~m}+1}$

Approximating $g_{2 m+\frac{1}{2}}$ by $\frac{3}{8} \mathrm{~g}_{2 \mathrm{~m}}+\frac{3}{4} \mathrm{~g}_{2 \mathrm{~m}+1}-\frac{1}{8} \mathrm{~g}_{2 \mathrm{~m}+2}$ and

inserting this into (19) yields a set of two linear equations for $g_{2 m+1}$ and $g_{2 m+2}$.

It follows from results by $\operatorname{Linz}^{[19]}$ that for fixed $y$ so that $n h=y$, the solution satisfies

$$
\left|g_{n}-g(y)\right|=O\left(h^{4}\right)
$$

provided $g$ is four times continuously

differentiable as is the case here by Theorem 2.1 in
Paulsen et $a l^{[23]}$. On the other hand, $\left|g_{2 m+2}-g_{2 m+1}\right|=O\left(h^{4}\right)$, as well, so there may be some fairly large oscillations. In (5), since $a_{2}=1$, we have $\alpha=\frac{1}{1-g_{a_{1}}(\infty)}$. To approximate $g_{a_{1}}(\infty)$, an upper bound $\bar{y}$ is chosen. Then to nullify the effect of the oscillations, we let $g_{a_{1}}(\infty)$ equal the average

$g_{a_{1}}(\infty)=\frac{1}{1000} \sum_{k=n_{0}-999}^{n_{0}} g_{k}$,

Where, $n_{0}=\frac{\bar{y}}{h}$. The constant $\bar{y}$ must be large enough so that $\psi(\bar{y}-999 h)$ is virtually equal to 1 . With this value of $g_{a_{1}}(\infty)$, the numerically calculated ruin probability becomes

$$
\psi(y)=\alpha g_{a_{1}}(y)+1-\alpha .
$$

Dickson and Waters ${ }^{[24]}$ presented several numerical methods for this case, but without any effort to analyse the error rate. Of all the methods they discuss, the one that is closest to ours is a method by Sundt and Teugels ${ }^{[16]}$. They showed that it performs very well, although it is clear from the algorithm of their method that it is of order no higher than 2. The results i show that the block-by-block method performs extremely well.

\section{NUMERICAL RESULTS}

We now report some numerical results obtained using the method described earlier.

For a given $y, \psi_{h}(y)$ is the calculated ruin probability when a stepsize $h$ is used. Since $\psi_{h}(y)$ is known, we compute the percentage relative error

$D_{h}(y)=\left|100 \cdot \frac{\psi_{h}(y)-\psi(y)}{\psi(y)}\right|$.

The implementation of the method we described earlier was done using FORTRAN and taking advantage of the Double Precision feature to get satisfactory accuracy. Of course slower programs like Splus, R, Gauss, Matlab, Maple or Mathematica could have been used, but at the expense of considerably longer computing time.

The process parameters $p=3, \lambda=2, r=0.05$ are used and $S$ is assumed to be exponentially distributed with expectation 2 . The true ruin probability was found way back by Segerdahl ${ }^{[5]}$ and later reappearing in many papers. The exact ruin probability is calculated to a high degree of accuracy from Segerdahl's formula using the Splus program integrate. Tables 1, 2 and 3 show that 
we can achieve the same results irrespective of what a1 we choose. The value of (0) is very accurate! As expected, the smaller the step size $h$ the better the results. This is the case as shown in Table 1-3 compared to the results in Table 4 . Note that the stepsize in Table 4 is 10 times that in Table 1-3.

\begin{tabular}{|c|c|c|c|}
\hline Ie & \multicolumn{3}{|c|}{$\begin{array}{l}\text { Ruin } \\
\mathrm{a}_{1}=0.300000, \mathrm{~g}_{\mathrm{a}_{1}}(\infty)=-47.580706, \alpha=0.020584\end{array}$} \\
\hline$y$ & $\psi(y)$ & $\psi_{0.01}(y)$ & $D_{0.01}(y)$ \\
\hline 0 & 0.985591 & 0.985591 & 0.000000 \\
\hline 10 & 0.806240 & 0.806504 & 0.032765 \\
\hline 20 & 0.494963 & 0.495288 & 0.065715 \\
\hline 30 & 0.215339 & 0.215556 & 0.100625 \\
\hline 40 & 0.066726 & 0.066815 & 0.133259 \\
\hline 50 & 0.015188 & 0.015213 & 0.162322 \\
\hline 60 & 0.002633 & 0.002638 & 0.187962 \\
\hline 70 & 0.000360 & 0.000361 & 0.209072 \\
\hline 80 & 0.000040 & 0.000040 & 0.218223 \\
\hline 90 & 0.000004 & 0.000004 & 0.255316 \\
\hline
\end{tabular}

Table 2: Ruin probabilities for $\mathrm{a}_{1}=0.500000, \mathrm{~g}_{\mathrm{a}_{1}}(\infty)=-33.700504, \alpha=0.028818$

\begin{tabular}{llll}
\hline$y$ & $\psi(y)$ & $\psi_{0.01}(y)$ & $D_{0.01}(y)$ \\
\hline 0 & 0.985591 & 0.985591 & 0.000000 \\
10 & 0.806240 & 0.806504 & 0.032765 \\
20 & 0.494963 & 0.495288 & 0.065715 \\
30 & 0.215339 & 0.215556 & 0.100624 \\
40 & 0.066726 & 0.066815 & 0.133258 \\
50 & 0.015188 & 0.015213 & 0.162326 \\
60 & 0.002633 & 0.002638 & 0.187960 \\
70 & 0.000360 & 0.000361 & 0.208983 \\
80 & 0.000040 & 0.000040 & 0.217341 \\
90 & 0.000004 & 0.000004 & 0.265045 \\
\hline
\end{tabular}

Table 3: Ruin probabilities for $\mathrm{a}_{1}=0.700000, \mathrm{~g}_{\mathrm{a}_{1}}(\infty)=-19.820302, \alpha=0.048030$

\begin{tabular}{llll}
\hline$y$ & $\psi(y)$ & $\psi_{0.01}(y)$ & $D_{0.01}(y)$ \\
\hline 0 & 0.985591 & 0.985591 & 0.000000 \\
10 & 0.806240 & 0.806504 & 0.032765 \\
20 & 0.494963 & 0.495288 & 0.065715 \\
30 & 0.215339 & 0.215556 & 0.100625 \\
40 & 0.066726 & 0.066815 & 0.133259 \\
50 & 0.015188 & 0.015213 & 0.162325 \\
60 & 0.002633 & 0.002638 & 0.187954 \\
70 & 0.000360 & 0.000361 & 0.209159 \\
80 & 0.000040 & 0.000040 & 0.218783 \\
90 & 0.000004 & 0.000004 & 0.249504 \\
\hline
\end{tabular}

Table 4: Ruin probabilities for $\mathrm{a}_{1}=0.300000, \mathrm{~g}_{\mathrm{a}_{1}}(\infty)=-47.580743, \alpha=0.020584$

\begin{tabular}{llcc}
\hline$y$ & $\psi(\mathrm{y})$ & $\psi_{0.1}(\mathrm{y})$ & $\mathrm{D}_{0.1}(\mathrm{y})$ \\
\hline 0 & 0.985591 & 0.985591 & 0.000001 \\
10 & 0.806240 & 0.808875 & 0.326810 \\
20 & 0.494963 & 0.498218 & 0.657524 \\
30 & 0.215339 & 0.217513 & 1.009289 \\
40 & 0.066726 & 0.067620 & 1.339229 \\
50 & 0.015188 & 0.015436 & 1.634071 \\
60 & 0.002633 & 0.002683 & 1.893354 \\
70 & 0.000360 & 0.000367 & 2.119934 \\
80 & 0.000040 & 0.000041 & 2.327116 \\
90 & 0.000004 & 0.000004 & 2.611787 \\
\hline
\end{tabular}

\section{CONCLUSION}

We have been able to use a numerical method which is simple and straight forward to calculate ultimate ruin probabilities under interest force. The results indicate the quality of our numerical method. An extension of this problem is to allow a diffusion in (1) thus

$$
Y_{t}=p t+\sigma_{P} W_{P, t}-\sum_{i=1}^{N_{t}} S_{i} ; \quad t \geq 0 .
$$

With another diffusion, the return on investments process will no longer be $R_{t}=r t$ but $R_{t}=r t+\sigma_{R} W_{R, t} ; \quad t \geq 0$.

For a detailed description of such a model, see e.g Paulsen et al. ${ }^{[23]}$. The same method is likely to work once the corresponding Volterra equation is obtained. The task will be to identify the right kernel and the forcing function to use in the FORTRAN program. Also, apart from the exponential distribution, other distributions can be used. In the event that the distribution is difficult to achieve analytically, Simpson's rule can be employed. Such an approach was used in Paulsen et al. ${ }^{[23]}$.

\section{ACKNOWLEDGEMENTS}

The authors extend their gratitude to NUFU project 33/02 and Carnegie Project under Carnegie/Mak/2002/0010 for the financial support and anonymous referees for the valuable comments.

\section{REFERENCES}

1. Lundberg, F., 1903. I. Approximerad Framställning av Sannolikhetsfunktionen. II. Återförsäkering av Kollektivrisker. Almqvist and Wiksell, Uppsala.

2. Cramer, H., 1930. On the Mathematical Theory of Risk. Skandia Jubilee Volume, Stockholm.

3. Cramér, H., 1955. Collective Risk Theory. Skandia Jubilee Volume, Stockholm.

4. Segerdahl, C.O., 1939. On homogeneous random processes and collective risk theory. Thesis. Stockholm. (Printed by Almqvist and Wiksell, Uppsala).

5. Segerdahl, C.O., 1942. über einige risikotheoretische Fragestellungen. Skandinavisk Aktuartidsskrift, 25: 43-83.

6. Andersen, E.S., 1957. On the collective theory of risk in case of contagion between the claims. Trans. XVth Intl. Cong. Actuaries, New York II, pp: 219-229.

7. Davidson, A., 1969. On the ruin problem in the collective theory of risk under the assumption of variable safety loading. Scand. Actuarial J., Suppl., The Filip Lundberg Symp., pp: 70-83. 
8. Thorin, O., 1970. Some remarks on the ruin problem in case the epochs of claims form a renewal process. Scand. Actuarial J., pp: 29-50.

9. Wikstad, N., 1971. Exemplification of ruin probabilities. Astin Bull., 6: 147-152.

10. Gerber, H.U., 1973. Martingales in risk theory. Mitteilungen der Vereinigung Schweizer Versicherungsmatematiker, 73: 205-216.

11. Harrison, J.M., 1977. Ruin problems with compounding assets. Stoch. Proc. Appl., 5: 67-79.

12. De Vylder, F., 1977. Martingales and ruin in a dynamical risk process. Scand. Actuarial J., pp: 217-225.

13. Bühlmann, J., 1970. Mathematical Methods in Risk Theory. Springer Verlag, Heidelberg.

14. Paulsen, J. and H.K. Gjessing, 1997. Ruin theory with stochastic return on investments. Adv. Appl. Prob., 29: 965-985.

15. Paulsen, J., 1998. Sharp conditions for certain ruin in a risk process with stochastic return on investments. Stoch. Proc. and their Appl., 75: 135148.

16. Sundt, B. and J. Teugels, 1995. Ruin estimates under interest force. Insurance: Math and Econ., 16: 7-22.

17. Asmussen, S. and H.M. Nielsen, 1995. Ruin probabilities via local adjustment coefficients. J. Appl. Prob., 29: 965-985.
18. Paulsen, J. and B.N. Rasmussen, 2003. Simulating ruin probabilities for a class of semimartingales by importance smapling methods. Scand. Actuarial J., pp: 178-216.

19. Linz, P., 1985. Analytical and Numerical Methods for Volterra Equations. SIAM Studies in Applied Mathematics, Philadelphia.

20. Moiseiwitsch, B.L., 1977. Integral Equations. London: Longman.

21. Widder, D.V., 1971. An Introduction to Transform Theory. Academic Press, New York.

22. Press, W.H., S.A. Teukolsky, W.T. Vetterling and B.P. Flannery, 1992. Numerical Recipes in FORTRAN - The Art of Scientific Computing. Sec. Edn. Cambridge University Press.

23. Paulsen, J., J. Kasozi and A. Steigen, 2005. A numerical method to find the probability of ultimate ruin in the classical risk model with stochastic return on investments. Insurance: Math and Econ., 36: 399-420.

24. Dickson, D.C.M. and H.R. Waters, 1999. Ruin probabilities with compounding assets. Insurance: Math and Econ., 25: 49-62. 